RESEARCH ETHICS

\title{
Randomisation in trials: do potential trial participants understand it and find it acceptable?
}

\author{
C Kerr, E Robinson, A Stevens, D Braunholtz, S Edwards, R Lilford
}

J Med Ethics 2004;30:80-84

See end of article for authors' affiliations

.....................

Correspondence to: Cicely Kerr, Research Fellow, Department of Psychology, Keele University, Staffordshire, ST5 5BG, UK; c.e.p.kerr@keele.ac.uk

Revised version received 18 July 2002

Accepted for publication

26 January 2003

\begin{abstract}
Objective: To examine lay persons' ability to identify methods of random allocation and their acceptability of using methods of random allocation in a clinical trial context.

Design: Leaflets containing hypothetical medical, non-medical, and clinical trial scenarios involving random allocation, using material from guidelines for trial information leaflets.

Setting and participants: Adults attending further education colleges $(n=130)$, covering a wide range of ages, occupations, and levels of education.

Main measures: Judgements of whether each of five methods of allocation to two groups was random in a medical or non-medical scenario. Judgements of whether these allocation methods were acceptable in a randomised clinical trial scenario, with or without a scientific justification for randomisation.

Results: The majority of our group of participants judged correctly that allowing people their preference was not random, and that the following were random: using a computer with no information about the individual (recommended wording for MREC trial leaflets), tossing a coin, drawing a name out of a hat. Judgements were split over allocating people in turn (not a random allocation method but shares features with randomisation). Judgements were no different in medical and non-medical scenarios. Few of the correctly identified random methods were judged to be acceptable in a clinical trial scenario. Inclusion of a scientific justification for randomising significantly increased the acceptability of only one random method: allocation by computer.

Conclusions: Current UK guidelines' recommended description of random allocation by computer seems warranted. However, while potential trial participants may understand what random allocation means, they may find it unacceptable unless offered an acceptable justification for its use.
\end{abstract}

$\mathrm{R}$ andomised controlled trials (RCTs) play a central role in modern medical advances, because random allocation of patients to treatment arms is widely considered to be the best way of achieving results that genuinely increase our knowledge about treatment effectiveness. Random allocation involves an equal chance of being allocated to any one of the treatment arms. This is ethically justified when there are no convincing grounds for supposing that any patient would be advantaged or disadvantaged if allocated to one treatment arm rather than another (collective equipoise). ${ }^{1}$ Millions of patients worldwide agree to participate in RCTs, and each of them should understand and accept the procedures involved. Research suggests, however, that this ideal is not achieved in practice. A systematic review by Edwards et al identified a substantial amount of evidence that participants in RCTs often fail to understand that their treatment was selected at random from among those under comparison. ${ }^{2}$ Results from qualitative studies suggest that participants often struggle to accept randomisation in their clinical trial. ${ }^{3}{ }^{4}$ This raises concern that their consent may not be adequately informed.

Potential trial participants may bring little prior knowledge about trial design, and are likely to be taken by surprise when informed about random allocation. Ellis et al found that 31\% of a sample of outpatients who were not trial participants were unaware that treatment is allocated by chance in a randomised trial. ${ }^{5}$ Furthermore $74 \%$ thought that the doctor would ensure that they received the best of the treatments offered in a randomised trial. Similarly, Appelbaum et al argued that patients commonly hold a "therapeutic misconception" that every aspect of a clinical trial has been designed to benefit themselves. ${ }^{6}$ Other researchers have concluded the problem is connected to the term itself. In a survey of terms commonly used in clinical trial consent forms
Waggoner et al found that only $22 \%$ of a general public sample knew the word "randomly", and only $4 \%$ of those without higher education knew the meaning of the word, the authors suggested using "by chance" or "by the flip of a coin" instead. ${ }^{7}$ In contrast to the conclusion that random allocation might have no meaning to potential trial participants, in Featherstone and Donovan's qualitative study they reported that patients who had participated in a clinical trial interpreted "at random" to mean without purpose. ${ }^{3}$ To date no systematic exploration of lay understanding of random allocation used in clinical trial or other contexts has been carried out.

UK research ethics committees require researchers to prepare written information for patients approached to participate in the trial, which covers all important aspects of the trial judged to be necessary for informed consent. While it is also routine for trial information to be presented orally to participants by clinicians or researchers, only the written information is available for scrutiny and approval by research ethics committees. Furthermore, studies in which researchers or clinicians were asked about the content of their oral consent discussions with patients suggest that trial design receives relatively little discussion. One study found that researchers rarely planned meaningful discussion beyond study purpose and procedures. ${ }^{8}$ Another found that in a consent discussion clinicians emphasised aspects of the trial that they expected a patient to understand easily, and did not emphasise issues that they expected patients to have more difficulty understanding, which included study design, randomisation, and the selection procedure. ${ }^{9}$

The apparent lay lack of knowledge about trial design, the possible holding of a "therapeutic misconception", and the likely brevity of discussion of trial design at consent, leave 
trial information leaflets with a heavy burden of responsibility for ensuring that consent to participate is adequately obtained. In the past, trial leaflets may have varied greatly in the way they informed patients about random allocation. Recently however, UK guidelines aimed at improving the content and readability of trial information have been incorporated into Multicentre Research Ethics Committees (MREC) application procedures. ${ }^{10}$ These guidelines for good clinical practice were produced by a working party which consulted with relevant experts and representatives of patient groups. For random allocation the guidelines advocate the description: "The groups are selected by a computer which has no information about the individual".

The recommended wording in these guidelines does not include an explanation or justification for the use of randomisation in clinical trials. An earlier set of noncompulsory guidelines produced by Consumers for Ethics in Research (CERES), ${ }^{11}$ an independent forum advocating the involvement of health service users in all stages of research, do include such a justification: "Randomised trials are the most exact and fair way to test which treatments work best. They are less likely to have, for example, people who are older or sicker in any one group. Each year thousands of people take part in them." These guidelines were informed by research carried out with women diagnosed with breast cancer, and produced in consultation with the CERES membership. Although both sets of guidelines were developed through a thorough consultation process, the extent to which they are understood by potential trial participants is at present unclear. One aim of this study was to begin to find out what background knowledge potential trial participants might draw upon when they are faced with information that allocation to treatment arms will be at random.

How can we judge whether or not potential trial participants have an adequate understanding of what random allocation means? One possible criterion is that participants must demonstrate explicit understanding by giving a verbal definition. Another possible criterion is that participants must demonstrate a working understanding by identifying examples of random and non-random allocation methods. If the participants' overall pattern of responses matches the pattern given by experts whose understanding is not in doubt, then we can infer that the participants have an adequate working understanding of random allocation. This kind of distinction between explicit and working (or implicit) knowledge is widely used in cognitive psychology-for example, in connection with people's ability to use syntactical rules without being able to state what those rules are. We argue that working understanding of random methods of allocation, rather than explicit understanding, is needed to make an informed decision whether or not to participate in a RCT.

Like previous published studies, ${ }^{12}$ we have used hypothetical trial scenarios with a non-clinical sample. As we are investigating pre-existing lay understanding of a concept common to many trials it is useful to be able to examine these free from the ethical constraints, specific individual concerns, and unique details of a specific clinical situation. Other studies using this technique have been criticised for generalising from judgements made concerning hypothetical trials to patients' judgements about trial entry. The latter group are clearly likely to have additional concerns specific to their particular circumstances that would play a role in their decision making. However we have been careful not to ask our non-clinical sample to consider whether they would be willing to participate in a hypothetical trial. Instead we set members of the public a task which requires them to draw on their pre-existing working understanding of random allocation. We ask them to judge whether or not each of a set of allocation methods is random. The random methods in the set include the description of randomisation recommended by the MREC guidelines, "by a computer which has no information about the individual". We include alternate allocation, a method which is not random and is rarely used in clinical trials, but which is a possible alternative to random allocation as it can abolish selection bias equally well if applied strictly. ${ }^{13}$ We elicit judgements about random and non-random methods of allocation in two different scenarios, neither involving a clinical trial, one medical (allocations to one of two consultants) and the other non-medical (allocations to one of two class outings). This allows us to explore the possibility that judgements are influenced by the belief that decisions in medical situations are generally made on therapeutic grounds.

Somebody who fully understands what random allocation involves may or may not consider it an acceptable procedure to use in a particular context. In addition to judging whether various methods of allocation would be random, our participants judge whether each method would be acceptable in a hypothetical randomised clinical trial scenario. We use the justification given in the CERES guidelines with half of the participants to assess whether its inclusion increases the acceptability of the various methods of random allocation. Like random allocation, alternate allocation does not allow patients or their doctors to select a treatment. It is interesting therefore to assess how acceptable it might be in the context of a clinical trial.

\section{METHODS}

\section{Participants}

The general public sample was made up of 130 adult students (at least 18 years of age) enrolled in further education and leisure courses at two colleges based at five sites in North Staffordshire. Twelve of these people also participated individually in interviews.

\section{Design and materials}

Each participant read a leaflet which first described one of two brief hypothetical scenarios, either:

1. The class trip scenario: Imagine that as part of a course everyone is entitled to go on a free trip either to Birmingham or Barcelona. However, there is only funding for about half to go to each place. The organisers decide to divide the class into two groups by chance, this means putting people into either the Birmingham or the Barcelona group at random. OR

2. The consultant scenario: Imagine that a doctor has many patients with back pain. He can refer these patients to one of two consultants. One consultant is based at the local hospital, the other is based at a hospital 30 miles

\section{Study aims}

To assess:

- Participants' judgements of whether or not allocation methods are random in two hypothetical, non-trial scenarios, one medical and the other non-medical.

- The acceptability in a hypothetical randomised clinical trial scenario of allocation methods previously identified by participants as random.

- The effect on acceptability judgements of providing the CERES justification for the use of random allocation in clinical trials. 
away. He can only refer about half of his patients to each consultant, so the doctor decides to divide the patients into two groups by chance, this means putting people into either the local consultant or the distant consultant group at random.

Immediately below the scenario there followed a list of five methods of allocating people to the two groups, and participants were asked to judge each as random or not random. The methods were:

A. Select the groups by a computer which has no information about each individual.

B. For each person toss a coin, heads means Birmingham/ near consultant, tails means Barcelona/far consultant.

C. Put printed slips of paper, an equal number of each, into a hat and for each person take a slip of paper out of the hat.

D. Ask each person which they prefer.

E. Allocate each person in turn as they arrive.

The methods were listed in three different orders (varied between leaflets): DBEAC, ACDBE, or CDAEB. After the list of allocation methods, leaflets contained a further scenario, giving a description of a hypothetical clinical trial: Imagine you are asked to take part in some medical research to compare two treatments. Imagine doctors currently know that both treatments help, but do not know which treatment is best. The research involves giving you one of the two treatments at random.

Half of participants were given only that description. The other half of the participants were told that medical research often requires participants to be allocated to treatment at random, and the justification of randomisation taken from the CERES guidelines:

"Randomised trials are the most exact and fair way to test
which treatments work best. They are less likely to have,
for example, people who are older or sicker in any one
group. Each year thousands of people take part in them."

The five methods of allocation were listed again and participants judged whether each would or would not be acceptable in the trial. On the basis of pilot work we decided to leave it unspecified as to whether acceptability was to the research, to the participant, or to both. This design produced four different leaflets:

1. Class trip scenario, then trial scenario with no justification for randomisation.

2. Consultant scenario, then trial scenario with no justification for randomisation.

3. Class trip scenario, then trial scenario with CERES justification for randomisation.

4. Consultant scenario, then trial scenario with CERES justification for randomisation.

\section{Procedure}

Random number tables were used to decide which leaflet would be given first (for example, leaflet four) and thereafter they were distributed in sequence (for example, 4123412341 etc) to the students as they had chosen to sit that day. This ensured that all leaflets were equally used, and that participants did not receive the same leaflet as their neighbours. Students were informed of the type of task they were being asked to complete, of those responsible for the NHS funded research, and that participation was voluntary. Average class size was nine students (range 5-16). Students completed the leaflets without discussion. The response rate for all students approached was over $95 \%$.

After the class as a whole had finished, volunteers (given a $£ 5$ voucher for their extra time) participated in audio taped individual discussions with the researcher, explaining their judgements. These interviews were carried out to check the basis of individual participants' judgements of random allocation methods and their reasons for acceptability responses. No more than four students from any one class were interviewed, and a total of 12 individual interviews were carried out. Following completion of interviews each class was debriefed as to the aims of the research.

\section{RESULTS}

\section{Sample characteristics}

The strategy of approaching as many adult education classes as possible in each of the colleges produced a sample of 130 people of various ages, occupations, and educational levels. Ages of participants ranged from 18 to 70 years with a mean age for the sample of 32.4 years (SD 10.1). There was a balance of occupations in the sample including housewives, retired people, students, or those with no occupation (26.2\%); those in manual, semi-skilled, or unskilled occupations $(31.5 \%)$, and those in skilled non-manual, managerial, or professional occupations (33.8\%). Most participants were not educated beyond GCSE or O level qualifications, usually taken at 16 years $(62.3 \%)$ with $12.3 \%$ reporting no educational qualifications. The sample contained $66.9 \%$ females.

\section{Judgements of whether or not allocation methods were random}

Table 1 shows, separately for participants given the class trip or the consultant scenario, the numbers who judged each method as random or not. Confidence intervals (CI) at the 95\% level were calculated for the percentage of participants judging a certain way. ${ }^{14}$ Where both the upper and lower limits of confidence intervals lie above $50 \%$ we conclude that a significant majority of the group gave that judgement; where both limits of confidence intervals lie below 50\% we conclude that the majority of the group did not give that judgement. If the confidence interval spans $50 \%$, we conclude that neither judgement was given by the majority of the group.

The results in table 1 show that most people, regardless of whether they read the consultant (medical) or class trip (non-medical) scenario, correctly judged "ask which prefer" as not random, and "computer", "toss coin", and "draw out of hat" as random. As a group, people had no consistent view (regardless of scenario considered) as to whether "allocate in turn" was random. As intended the individual interviews provided a check of the basis of these judgements and supported the validity of these findings (although the number of interviews is insufficient to warrant more in depth analysis). In the main participants reported that they based their judgements of random allocation on meaningful criteria such as the unpredictability of the result of the allocation $(\mathrm{n}=3)$ ("it could be any"), the effectiveness of the method in achieving roughly half of participants in each group $(n=5)$ ("you would be able to get a balance"), avoiding bias or influence on the allocation $(\mathrm{n}=3)$ ("nobody's got the influence to make the decision"), lack of choice $(n=2)$ ("because you wouldn't have any choice whatsoever"), not taking into account individual characteristics or circumstances $(n=2)$ ("half of them go there, half of them go there, you've got no information about them"), and luck $(\mathrm{n}=2)$ ("the luck of the draw"). 
Table 1 Judgements of methods of allocation as random or not random

\begin{tabular}{|c|c|c|c|c|c|c|c|c|}
\hline \multirow[b]{4}{*}{ Method of allocation } & \multicolumn{6}{|c|}{ Scenario given } & & \\
\hline & \multicolumn{3}{|l|}{ Class trip } & \multicolumn{3}{|l|}{ Consultant } & \multicolumn{2}{|c|}{ Correct totals } \\
\hline & \multicolumn{2}{|l|}{ Random } & \multirow{2}{*}{$\begin{array}{l}\text { Not random } \\
\mathbf{n}(\%)\end{array}$} & \multicolumn{2}{|l|}{ Random } & \multirow{2}{*}{$\frac{\text { Not random }}{\mathrm{n}(\%)}$} & \multirow[b]{2}{*}{ Random } & \multirow[b]{2}{*}{ Not randon } \\
\hline & n (\%) & $95 \% \mathrm{Cl}$ & & n (\%) & $95 \% \mathrm{Cl}$ & & & \\
\hline Computer & 55 (85.9) & 75.3 to 92.4 & $9(14.1)^{*}$ & $55(87.3)$ & 76.9 to 93.4 & $8(12.7) \dagger$ & 110 & $17 \ddagger$ \\
\hline Toss coin & $48(75.0)$ & 63.2 to 84.0 & $16(25.0)^{*}$ & $48(77.4)$ & 65.6 to 86.0 & $14(22.6) \ddagger$ & 96 & $30 \S$ \\
\hline Draw out of hat & $58(92.1)$ & 82.7 to 96.6 & $5(7.9) \dagger$ & $46(73.0)$ & 61.0 to 82.4 & $17(27.0) \dagger$ & 104 & $22 \S$ \\
\hline Ask which prefer & $5(8.1)$ & 3.5 to 17.5 & $57(91.9) \ddagger$ & $14(22.6)$ & 14.0 to 34.4 & 48 (77.4)‡ & 19 & $105^{\top}$ \\
\hline Allocate in turn & $30(46.9)$ & 35.2 to 58.9 & $34(53.1)^{*}$ & $34(54.0)$ & 41.8 to 65.7 & $29(46.0) \dagger$ & 64 & $63 \ddagger$ \\
\hline
\end{tabular}

*Missing data, $\mathrm{n}=1$; †missing data, $\mathrm{n}=2$; ¥missing data, $\mathrm{n}=3$; §missing data, $\mathrm{n}=4$; \% missing data, $\mathrm{n}=6$.

The bold correct totals form basis for table 2 .

$\mathrm{Cl}$, confidence interval.

\section{Acceptability of procedures in clinical trial context}

Since the two scenarios showed similar patterns of judgements, we combined responses in the two scenarios for analysis of the judgements of acceptability. Table 2 shows the acceptability of the random methods (computer, toss coin, draw out of hat) and the main non-random method (ask which prefer) in a randomised clinical trial among participants who correctly judged the random/non-random nature of the allocation method in the first scenario. The results are split by whether or not the participants were given leaflets with the CERES justification for randomisation. Confidence intervals at the $95 \%$ level are given for the percentage of participants, with or without the justification, who judged each allocation method to be acceptable. These were interpreted as before. Confidence intervals for the difference in these percentages are also given: a CI not spanning zero indicates that the presence/absence of the justification probably caused a differential response.

The percentages (of those who identified the method as random) judging "toss a coin" and "draw out of a hat" as acceptable are very similar to each other and did not depend on whether the justification was given or not. A significant majority judged each of these methods to be unacceptable. The other random allocation method, computer, was more likely to be judged acceptable by those receiving the justification, though still in neither case did a significant majority judge it acceptable. "Ask which prefer" was judged acceptable by a somewhat higher percentage of those who were not given the justification, though this may have been due to chance.

The acceptability of "allocate in turn" is not included in table 2 due to its status as a non-random method which nevertheless potentially achieves the reduction in selection bias that randomisation aims for. It is not therefore informative to consider acceptability judgements in light of whether or not it was judged random.
Allocate in turn was judged acceptable by 18 of those who received no justification for randomisation $(28.6 \%$, 95\% CI 18.9 to 40.7 , missing data $n=3$ ) and by 18 of those who received the CERES justification for randomisation $(29.0 \%$, $95 \%$ CI 19.2 to 41.3 , missing data $n=2$ ).

The interviews contained considerable discussion of why certain methods of allocation were acceptable and others not. Computer allocation when found to be acceptable was so because it has no personal information about you, it is the least open to bias and it is more removed (from the actions of the doctor) than other random methods. Some of the other random methods were considered unprofessional or not how participants would want to think their treatment had been decided. This excerpt from the fifth participant interviewed sums up many of these points:

"I wouldn't want to think that my doctor had decided what I was going to have by flipping a coin, although it is a random way of doing, and the same with drawing a name out of a hat and yet I have no problem with a computer doing it. I think it's just the way it's, I don't know, a computer is a little more distant, again it's not the doctor doing it, he's allowing the computer to do it."

\section{DISCUSSION}

When asked to judge whether or not the allocation methods were random the majority of participants were able to do so correctly, apparently based on relevant criteria, and irrespective of whether the setting was medical or non-medical. Not surprisingly, participants as a group were divided as to whether allocating in turn was a random method: it could produce unbiased samples despite being a systematic method.

Might our method of eliciting working understanding yield false positives? Might participants give correct judgements

Table 2 Judgements of acceptability of methods of allocation in a clinical trial context by participant who correctly judged the methods as random or non-random

\begin{tabular}{|c|c|c|c|c|c|c|c|c|}
\hline \multirow{3}{*}{$\begin{array}{l}\text { Method of } \\
\text { allocation }\end{array}$} & \multirow{3}{*}{$\begin{array}{l}\text { Correctly } \\
\text { judging as } \\
\text { random (n) }\end{array}$} & \multicolumn{3}{|c|}{ No justification for randomisation } & \multicolumn{3}{|c|}{ CERES justification for randomisation } & \multirow[b]{3}{*}{ Difference $95 \% \mathrm{Cl}$} \\
\hline & & \multicolumn{2}{|l|}{ Acceptable } & \multirow{2}{*}{$\frac{\text { Not acceptable }}{\text { n (\%) }}$} & \multicolumn{2}{|l|}{ Acceptable } & \multirow{2}{*}{$\frac{\text { Not acceptable }}{n(\%)}$} & \\
\hline & & $\overline{n(\%)}$ & $95 \% \mathrm{Cl}$ & & $n(\%)$ & $95 \% \mathrm{Cl}$ & & \\
\hline Computer & 110 & $20(37.7)$ & 25.9 to 51.2 & $33(62.3)^{*}$ & $32(58.2)$ & 45.0 to 70.3 & $23(41.8)$ & -0.373 to -0.016 \\
\hline Toss coin & 96 & $13(27.7)$ & 16.9 to 41.8 & $34(72.3) \dagger$ & $15(32.6)$ & 20.9 to 47.0 & $31(67.4)$ & -0.229 to 0.134 \\
\hline Draw out of hat & 104 & $19(35.8)$ & 24.3 to 49.3 & $34(64.2)^{*}$ & $17(34.7)$ & 22.9 to 48.7 & $32(65.3)$ & -0.170 to 0.190 \\
\hline Ask which prefer & 106 & $39(75.0)$ & 61.8 to 84.8 & $13(25.0)$ & $32(60.4)$ & 46.9 to 72.4 & $21(39.6)$ & -0.032 to 0.312 \\
\hline
\end{tabular}

*Missing data $n=2 ;$ †missing data, $n=3$; applies to those who gave correct judgements of random allocation methods.

$\mathrm{Cl}$, confidence interval. 
despite really having a misconception of what random allocation involves? We cannot think of a single misconception which would produce correct judgements across several of the allocation methods. Perhaps participants drew on a set of different misconceptions for the different methods. One possibility is that participants thought that a computer might use attendance records to allocate people to one or other class trip, without seeing this as information about each individual. It might be possible to identify interpretations that would lead to false positives for each of the other random methods described, but the interviews did not reveal any. A more parsimonious conclusion is that the majority of participants shared our concept of random allocation.

Given that all participants were told that the clinical trial involved allocating treatments at random, it is interesting to note that the acceptability of the random methods was low among the majority of the sample who had correctly judged the methods as random. Allocate in turn, a non-random though scientifically appropriate method was also judged to be unacceptable by the majority of our participants. Earlier authors have concluded the term "random" is often not understood. ${ }^{7}$ We suggest that this simple explanation cannot account for the pattern of acceptability judgements in the current study. As argued above, our participants appeared to share our concept of random.

It is not clear why a large proportion of the people who correctly judged "ask which prefer" as not random, nevertheless saw it as an acceptable method of allocation in a randomised trial. One possibility is that these participants accepted that random allocation was to be used but interpreted "acceptable" as "acceptable to patients". Another possibility is that they rejected the idea that the trial should be randomised. Consistent with this rejection of randomisation, they picked a different allocation method which they thought more appropriate. One reason participants might have taken this approach could be that from their perspective random allocation had no purpose within a trial context, as implied by the lay meaning reported by patients in an earlier study. ${ }^{3}$ Results of other investigations suggest that members of the public see no scientific benefits of random allocation: ${ }^{15}$ people judged that doctors would be just as sure about which treatment was most effective whether allocation was at random or by doctor and patient choice. In the current investigation, the inclusion of the CERES wording to justify random allocation had little impact on participants' acceptability judgments. Maybe a more detailed explanation would help people recognise the scientific purpose behind random allocation in a trial context. It remains to be seen whether or not this would render random allocation more acceptable.

\section{CONCLUSION}

Our results support the current UK guidelines ${ }^{10}$ in their recommended description of randomisation "the groups are selected by a computer which has no information about the individual." Our results give no cause for concern that potential trial participants fail to understand what random allocation is, or that a computer can achieve it. In addition this was the lone random method that was more likely to be judged acceptable when coupled with the CERES scientific justification for randomisation. We found no evidence that other analogies were more easily identified as random. However the relatively low acceptability judgements do raise concerns that potential participants may need a more powerful justification for random allocation of treatments in clinical trials.

\section{Authors' affiliations}

C Kerr, E Robinson, Department of Psychology, Keele University, UK A Stevens, D Braunholtz, R Lilford, Department of Public Health and Epidemiology, The University of Birmingham, UK

$S$ Edwards, Centre for Ethics in Medicine, University of Bristol, UK

This study was supported by the methodology section of the National Health Technology Assessment (HTA) programme.

The views expressed in this paper are those of the authors and do not necessarily reflect those of the HTA.

Approval for this study was granted by the Keele Psychology Research Ethics Committee.

\section{REFERENCES}

1 Freedman B. Equipoise and the ethics of clinical research. N Engl J Med 1987;317:141-5.

2 Edwards SJL, Lilford RJ, Braunholtz DA, et al. Ethical issues in the design and conduct of randomised controlled trials. Health Technol Assess 1998;2:i-vi, $1-132$.

3 Featherstone K, Donovan JL. Random allocation or allocation at random? Patients' perspectives of participation in a randomised controlled trial. BMJ 1998;317:1177-80.

4 Featherstone K, Donovan JL. "Why don't they just tell me straight, why allocate it?" The struggle to make sense of participating in a randomised controlled trial. Soc Sci Med 2002;55:709-19.

5 Ellis PM, Dowsett SM, Butow PN, et al. Attitudes to randomized clinical trials amongst out-patients attending a medical oncology clinic. Health Expect 1999;2:33-43.

6 Appelbaum PS, Roth LH, Lidz CW, et al. False hopes and best data: consent to research and the therapeutic misconception. Hastings Cent Rep 1987;17:20-4

7 Waggoner WC, Mayo DM. Who understands? A survey of 25 words or phrases commonly used in proposed clinical research consent forms. IRB 1995; 17:6-9.

8 Titus SL, Keane MA. Do you understand? An ethical assessment of researchers' description of consenting process. J Clin Ethics 1996;7:60-8.

9 Verheggen FWSM, Jonkers R, Kok G. Patients' perceptions on informed consent and the quality of information disclosure in clinical trials. Patient Educ Couns 1996;29:137-53.

10 Central Office for Research Ethics Committees (COREC). Guidelines for researchers: patient information sheet and consent form. http:// www.corec.org.uk.

11 CERES Consumers for Ethics in Research. Spreading the word on research, or patient information: how can we get it better? London: CERES, 1994.

12 Corbett F, Oldham J, Lilford R. Offering patients entry in clinical trials: preliminary study of the views of prospective participants. J Med Ethics 1996;22:227-31.

13 Chalmers I. Controlled trials from history. http://www.jameslindlibrary.org.

14 Newcombe RG. Confidence intervals for proportions, differences between proportions and related quantities. University of Wales College of Medicine, Department of Medical Computing and Statistics. hittp://

www.archive.uwcm.ac.uk/uwcm/ms/Robert2.html [accessed 2002]

15 Robinson EJ, Kerr C, Stevens A, et al. Lay conceptions of the ethical and scientific justifications for random allocation in clinical trials. Soc Sci Med (in press). 